\title{
HIGH FREQUENCY SIGNAL IN GOLF DATA
}

\author{
R. A. GARCÍA ${ }^{1}$, P. L. PALLÉ ${ }^{2}$ AND THE GOLF TEAM ${ }^{\dagger}$ \\ ${ }^{1}$ SAp, DAPNIA/DSM, CE Saclay, 91191 France \\ ${ }^{2}$ Instituto de Astrofisica de Canarias, E-38205, Tenerife, Spain \\ ${ }^{\dagger}$ GOLF Team: P. Boumier, J. Charra, A.H. Gabriel, G. Grec, J.M. Robillot, \\ T. Roca Cortés, S. Turck-Chièze, R.K. Ulrich
}

\begin{abstract}
.
The analysis of $\approx 460$ days of high quality data provided by the GOLF experiment on board SOHO, has unambiguously revealed the presence of signal in the power spectrum in the region 5.8 to $7.5 \mathrm{mHz}$, well above the p-modes cut-off frequency. In this contribution, the observed structure of these fulldisk observations is presented. High frequency peaks (hereafter HFPs) well above the acoustic cutoff frequency of the solar p modes $(\approx 5.5 \mathrm{mHz})$ have been already observed in intermediate and high spatial resolution oscillation data (Duvall et al. 1991). The different interpretations of the observed pattern (Kumar 1994 and references therein; Rast and Gough 1995) agree that it provides direct information on the location and some characteristics of the source of the acoustic oscillations (see also Vorontsov et al. 1997).
\end{abstract}

\section{Observations}

The high frequency range of the GOLF velocity power spectrum has been analyzed using 460 days beginning on April 11, 1996. GOLF is a disk-integrated sunlight resonant scattering spectrophotometer flying on board the SOHO mission. In its actual configuration mode, it obtains the line of sight velocity of the integrated visible solar surface by measuring the Doppler shift in the blue line wings of the sodium doublet.

As the high frequency structures are not expected to be coherent over a time longer than several hours, 115 subseries of 4 days have been extracted from the GOLF time series and their power spectrum has been averaged. Individual spectra were calculated using average of FFTs and crossed-spectra between the two photomultipliers.

\section{Results}

- Providing enough statistics, HFPs are clearly visible and independent of the spectral 
technics (average of FFTs or crossed-spectra), the number of days averaged (410), degree of smoothing, and method of velocity calibration.

- 3 different regions have been observed (see figure 1):

1. $<5.2 \mathrm{mHz}$. Unresolved high $n$ p-mode envelopes (odd and even degree), separated $\sim 60$ to $80 \mu \mathrm{Hz}$ depending on the frequency interval.

2. $\sim 5.4-5.8 \mathrm{mHz}$. No signal. Acoustic cut off frequency region.

3. $>5.8 \mathrm{mHz}$. HFPs pattern with characteristics of pseudo-modes.

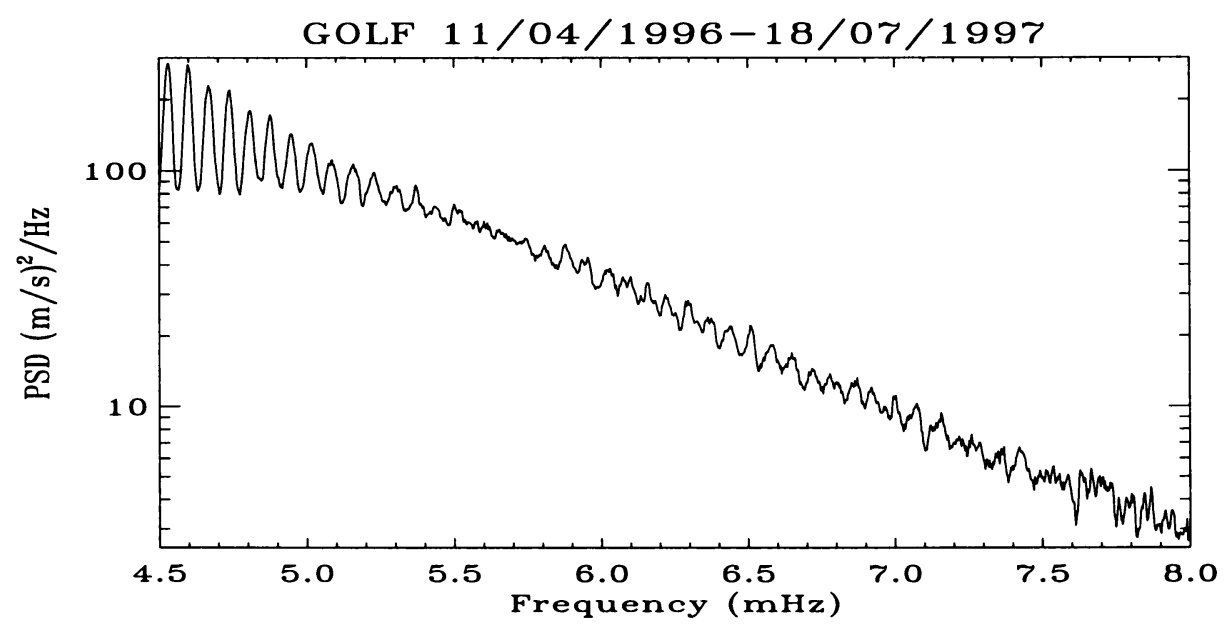

Figure 1. Average GOLF power spectrum of 115 Subseries of 4 days each smoothed with a boxcar of 5 points. A clear transition region between the $\mathrm{p}$ modes and the HFPs can be observed $\approx 5.5-5.8 \mathrm{mHz}$. The HFPs pattern are visible between 5.8 to $7.5 \mathrm{mHz}$.

- HFPs pattern consists on a unique series of broad (FWHM $\sim 28.89 \pm 2.00 \mu \mathrm{Hz}$ ) equally spaced peaks $(\sim 70.38 \pm 1.83 \mu \mathrm{Hz})$ that can be modelled with a simple damped sinusoid.

- Global Sun observations do not allow to see visible variations of the width or spacing of the peaks as a function of frequency.

- Neither is there a phase shift seen in the HFPs pattern between intensity and velocity, nor between the two intensities at different height of the solar atmosphere.

- Above $7.5 \mathrm{mHz}$, more data are required.

\section{References}

Duvall, T.L.Jr., Harvey, J.W., Jefferies, S.M. and Pomerantz M.A. (1991), ApJ Vol. 373, p. 308

Kumar, P.(1994), ApJ Vol. 428, p. 827

Rast, M.P. and Gough, D.O. (1995), GONG'94: Helio-and Astroseismology from the Earth and Space. A.S.P. Conference Series, R.K. Ulrich, E.J. Rhodes Jr. and W. Dappen. Ed., Vol. 76, p. 322

Vorontsov, S.V., Jefferies, S.M., Duvall, T.L.Jr. and Harvey, H.(1997), MNRAS Submitted 